No conflict-of-interest rules

\section{Washington}

THE US National Institutes of Health (NIH) last week abandoned plans to prepare guidelines aimed at preventing conflicts of interest in $\mathrm{NIH}$-sponsored research after receiving hundreds of critical comments on their proposals. In place of the guidelines, which would have required researchers to disclose in detail their personal financial interests as well as all non-federal sources of research support, NIH officials say they will propose rules that will be legally binding, but considerably smaller in scope.

Louis Sullivan, Secretary of the Department of Health and Human Services (HHS), says that "widespread concern" among researchers lay behind his decision to abandon the controversial proposals. "It is important that we do not impose on our scientific community regulatory burdens which may be unnecessary or counterproductive", he said.

The conflict-of-interest guidelines have been widely condemned by NIH-funded researchers since they were first proposed in September (see Nature 341, 173; 1989) after two years of pressure for regulation from the House subcommittee on human resources and intergovernmental relations, chaired by Representative Ted Weiss (Democrat, New York). Most NIH-funded institutions already have some sort of procedures to screen for conflicts of interest and researchers argue that further restrictions were unwarranted.

Because the guidelines would have applied not just to principal investigators but to "all persons who are in a position to have ... substantive control over [NIHfunded] research", compliance would have demanded enormous quantities of paperwork.

Institutions would have to scrutinize their researchers' financial interests and outside professional activities to ensure that their work would not be compromised by, for example, ownership of stock in pharmaceutical companies. Within the University of California alone, according to its director of research and public policy, Belle Cole, meeting the requirements could cost $\$ 10$ million a year.

The guidelines might not stand up to legal assault, according to Robert Charrow of the law firm Crowell and Moring. The proposed standards would dictate policy to institutions receiving NIH funding, he says, making it arguable that they would have the de facto status of formal 'rules', which must go through a long approval process involving several federal agencies, including HHS and the Office of Management and Budget.

With watered-down regulations, the NIH may be able both to circumvent a lengthy approval process and to deflect scientific protest. Current plans are to restrict the new regulations so that the total annual cost to all NIH-funded research institutions would be considerably less than $\$ 100$ million, says Katherine Bick, deputy director for extramural research at NIH.

Limiting the economic impact allows the proposed regulation to be called a 'minor rule', a bureaucratic distinction that can shorten the approval process by years by avoiding several layers of public comment. And looser regulations may be necessary to obtain the cooperation of the research community. "We want to ensure that anything of this sort is as unobtrusive as possible", she says.

Bick hopes to have proposals out by early summer. Although the comments received in response to the now-abandoned guidelines will help form the new rules, she expects to allow for new opportunities for public comment on the issue

According to Bick, there is only "anecdotal evidence" that a conflict-ofinterest problem exists. But Weiss may not be as charitable. "[we] are concerned that NIH appears to be backing down", says James Gottlieb, a Weiss aide. If NIH officials refuse to "act responsibly" and get a grip on the conflict of interest issue, he says, Weiss may introduce legislation to do it for them. And there is evidence that the situation is not as rosy as researchers may like to believe. In a study last year by the Acadia Institute, an academic think-tank in Bar Harbor, Maine, and three other organizations, 69 per cent of the surveyed institutions reported allegations of improper conflict of interest.

G. Chrlstopher Anderson

NEUTRINO ASTRONOMY

\title{
SNO ball starts rolling
}

\section{Washington}

THE Sudbury Neutrino Observatory (SNO), a \$52-million experiment that will investigate the Sun from the depths of a Canadian nickel mine, received final approval and $\$ 30.1$ million from the National Sciences and Engineering Research Council (NSERC) of Canada last week. With $\$ 6.5$ million from the project's home province of Ontario and $\$ 14.6$ million from the US Department of Energy already promised, the observatory is now waiting only for a final decision on $\$ 1$ million from the United Kingdom.

Construction will begin in the next few months, NSERC said. Located in a 10 storey cavern more than a mile underground, the facility will consist of a 14metre-tall acrylic tank containing 1,000 tonnes of heavy water - worth $\$ 300$ million - on loan from Atomic Energy of Canada (AEC). Surrounding the water will be 2,000 photomultiplier tubes. Special low-radiation construction materials and shielding to screen out spurious particles will make the cavern the lowest radioactivity site in the world.

The huge volume of heavy water will serve as a detector of neutrinos from the core of the Sun, as well as from any fortuitous nearby supernovae which would generate a pulse of high-energy neutrinos. Neutrinos react with deuterium nuclei in the heavy water, producing high-speed electrons and positrons which generate a burst of Cerenkov radiation. The Cerenkov light is then picked up by the array of photomultipliers. Similar neutrino detectors, one at Kamioka, Japan and another under Lake Erie, detected the neutrino burst from SN1987A. But the two older detectors have chambers filled with ordinary water, whose proton nuclei offer fewer possibilities for neutrino interaction than deuterium, and are therefore less efficient than $\mathrm{SNO}$ will be.

Arthur McDonald, director of SNO, hopes that the new facility will help to resolve the notorious solar neutrino problem. For many years, an underground detector at the Homestake mine in North Dakota has measured a solar neutrino flux of only about one-third that predicted by standard theory from the Sun's internal nuclear reactions. It remains unknown whether it is solar theory or neutrino physics that needs to be modified. A favoured but unproved explanation is that electron neutrinos emitted by the Sun transmute on their way here into other types, muon and tauon neutrinos, which the other detectors cannot pick up. SNO can detect all neutrino types, and so should resolve the problem. The apparatus is expected to detect several dozen neutrinos a day. But another SN1987A could generate as many as 1,000 neutrino detections in 10 seconds.

The loan of the heavy water and the donation of mine space by INCO Ltd saved the project some $\$ 390$ million, for which project planners can largely thank slower-than-expected sales of Canadian nuclear reactors. The 1,000 tonnes of heavy water comes from a stockpile AEC had intended to use in new reactors. If sales pick up, the observatory's days may be numbered; it has been promised the heavy water only for another nine years. But McDonald hopes that the basic questions on the Sun's neutrino flux will by then have been answered. After a fiveyear construction period, the observatory should accomplish most of its objectives within three years.

G. Christopher Anderson 